\title{
Wide Viewing Angle Multi-domain In-Plane Switching LCD
}

\author{
Ruibo Lu \\ Shin-Tson Wu \\ CREOL/College of Optics and Photonics, University of \\ Central Florida, Orlando, Florida, USA
}

\section{Qi Hong}

\section{Thomas X. Wu}

Department of Electrical and Computer Engineering, University of Central Florida, Orlando, Florida, USA

The bending angle effect on a multi-domain in-plane switching liquid crystal display (MD IPS LCD) using the chevron-shaped electrodes is investigated through a three-dimensional simulation. We find that when the bending angle of the electrodes increases from $10^{\circ}$ to $40^{\circ}$, the threshold voltage is lowered from $1.25 V_{\text {rms }}$ to $0.75 V_{\text {rms. }}$. With the optimized uniaxial compensation films, the viewing angle of the film-compensated MD IPS LCD is widened to $\pm 80^{\circ}$ viewing cone while keeping a 100:1 contrast ratio. These outstanding performances are expected to make impact to the flat panel display industry.

Keywords: compensation film; in-plane switching; liquid crystal display; wide viewing angle

\section{INTRODUCTION}

Fast response, wide viewing angle, and high contrast ratio are the main requirements for high quality transmissive LCDs, such as the large-screen LCD TVs and monitors [1]. Several operation modes, such as the multi-domain vertical alignment (MVA) [2], patterned vertical alignment (PVA) [3], advanced super view (ASV) [4] and in-plane

The authors are indebted to the financial support of Toppoly Optoelectronics Corporation (Taiwan).

Address correspondence to Ruibo Lu, CREOL/College of Optics and Photonics, University of Central Florida, Orlando, Florida 32816, USA. E-mail: rlu@mail.ucf.edu 
switching (IPS) [5-7], have been proposed to improve these technical issues. IPS LCD has the inherent wide viewing angle properties but it is still insufficient for the TV applications. To widen the viewing angle, the uniaxial or biaxial compensation films are usually adopted $[8,9]$. Typically, the viewing angle can be extended to $50: 1$ on the whole viewing cone with the biaxial compensation films.

In order to eliminate the color shift phenomenon in IPS mode, the multi-domain (MD) IPS LCD has been proposed using the zig-zag shaped electrodes or W-shaped electrodes, where the bending angle of the electrodes are usually at $2-15^{\circ}[10,11]$.

In this paper, we investigate the bending angle effect on a MD IPS using the chevron-shaped electrodes through a three-dimensional numerical simulation. The electro-optic properties of the MD IPS mode under different bending angles were characterized by the voltage-dependent transmittance (VT) curve, and iso contrast view angle contour. The uniaxial compensation films are optimized to widen the viewing angle of the MD IPS mode. We demonstrated that a MD IPS-LCD with enhanced view angle can be realized using a set of optimized positive C- and A-plates as the compensation films.

\section{LIGHT LEAKAGE IN LCD AND THE FILM COMPENSATION PRINCIPLE}

The normalized light transmittance $(\mathrm{T})$ through the LC medium under the crossed linear polarizers in a typical LCD can be described as [12]

$$
T=\sin ^{2}(2 \varphi) \sin ^{2}(\delta / 2) .
$$

where the angular dependent phase retardation of the LC medium at a certain wavelength, $\lambda$, is in the expression of

$$
\delta(\theta, \lambda)=\frac{2 \pi(d \cdot \Delta n)_{\mathrm{eff}}}{\lambda}
$$

and

$$
(d \cdot \Delta n)_{\mathrm{eff}}=\frac{d}{\cos \theta}\left\{\frac{n_{e} n_{o}}{\left[n_{o}^{2} \sin ^{2}(\theta \pm \phi)+n_{e}^{2} \cos ^{2}(\theta \pm \phi)\right]^{1 / 2}}-n_{o}\right\} .
$$

In Eqs. (2) and (3), $n_{o}$ and $n_{e}$ represent the ordinary and extraordinary refractive index, $\Delta n=n_{e}-n_{o}$ the birefringence, $n=\left(n_{e}+2 n_{o}\right) / 3$ the average refractive index of the LC material, $d$ is the LC cell gap, $\theta$ is incident angle on the LC medium and $\phi$ is the LC tilt angle.

It can be seen that if the incident angle $\theta$ is nonzero, then $(d \cdot \Delta n)_{\text {eff }} \neq 0$, i.e., the phase retardation from the LC medium exists 
and its value depends on the incident angle. This non vanishing phase retardation would cause light leakage from the crossed polarizers. This off-axis light leakage is detrimental to the contrast ratio under the oblique angles which, in turn, affects the viewing angle performance. Therefore, a crucial issue for enhancing contrast ratio over a wide viewing range is to eliminate the off-axis light leakage and minimize the dark state transmittance.

To minimize the off-axis light leakage, it is necessary to select proper optical phase compensation films to cancel the residual LC phase retardation at any oblique angles:

$$
(d \cdot \Delta n)_{\text {eff }}+(d \cdot \Delta n)_{\text {film }} \approx 0 .
$$

In addition, the dark state should remain as black as possible and insensitive to the viewing angle. These are the two basic criteria for implementing the phase compensation films. Different types of LCD are in need of different types of compensation films to get the ideal compensation effect [13].

\section{OPTICAL CALCULATIONS}

The director distributions of the LC molecules are obtained by a 3-D numerical model which combines the finite element method and finite difference method [14]. With the known steady state director distributions, the optical properties of the LC device can be solved using the extended Jones matrix method [15]. The LC layer is modeled as a stack of uniaxial homogeneous layers with the same structure as the previous model [16]. Here, we assume the reflections between interfaces are negligible. Therefore, the transmitted electric field is related to the incident electric field by

$$
\left[\begin{array}{l}
E_{x} \\
E_{y}
\end{array}\right]_{N+1}=\mathbf{J}\left[\begin{array}{l}
E_{x} \\
E_{y}
\end{array}\right]_{1}=\mathbf{J}_{\mathrm{Ext}} \mathbf{J}_{N} \mathbf{J}_{N-1} \cdots \mathbf{J}_{2} \mathbf{J}_{1} \mathbf{J}_{\mathrm{Ent}}\left[\begin{array}{l}
E_{x} \\
E_{y}
\end{array}\right]_{1},
$$

where $\mathbf{J}_{\mathrm{Ext}}$ and $\mathbf{J}_{\mathrm{Ent}}$ are the correction matrix considering the transmission losses in the air-LCD interface, which are given by

$$
\begin{aligned}
& \mathbf{J}_{\mathrm{Ent}}=\left[\begin{array}{cc}
\frac{2 \cos \theta_{p}}{\cos \theta_{p}+n_{p} \cos \theta_{k}} & 0 \\
0 & \frac{2 \cos \theta_{k}}{\cos \theta_{k}+n_{p} \cos \theta_{p}}
\end{array}\right], \\
& \mathbf{J}_{\mathrm{Ext}}=\left[\begin{array}{cc}
\frac{2 n_{p} \cos \theta_{k}}{\cos \theta_{p}+n_{p} \cos \theta_{k}} & 0 \\
0 & \frac{2 n_{p} \cos \theta_{p}}{\cos \theta_{k}+n_{p} \cos \theta_{p}}
\end{array}\right],
\end{aligned}
$$


Correspondingly, the overall optical transmittance is represented as

$$
t_{\mathrm{op}}=\frac{\left|E_{x, N+1}\right|^{2}+\cos ^{2}\left(\theta_{p}\right)\left|E_{y, N+1}\right|^{2}}{\left|E_{x, 1}\right|^{2}+\cos ^{2}\left(\theta_{p}\right)\left|E_{y, 1}\right|^{2}},
$$

where $n_{p}$ is the index of refraction of the polarizer, and $\theta_{p}$ is given by

$$
\theta_{p}=\sin ^{-1}\left(\frac{\sin \left(\theta_{k}\right)}{\operatorname{Re}\left(n_{e, p}+n_{o, p}\right) / 2}\right),
$$

in which $n_{e, p}$ and $n_{o, p}$ are the two refractive indices of the polarizer, and $\theta_{\mathrm{k}}$ is the azimuthal angle of the incident wavevector, $\mathbf{k}$.

The optical performance can be evaluated with the known optical transmittance $t_{\mathrm{op}}$. If the transmittance at the steady state with different applied voltage is solved, the voltage-dependent transmittance curve could be obtained. For a normally black LCD, the director distribution at the dark state is its initial director distribution. The transmittances of the dark state and the bright state at different viewing angles are solved separately, and then the contrast ratio at these viewing angles could be found. Therefore, the isocontrast contour bar at different spatial angles (including the polar angle and the azimuthal angle) can be plotted accordingly.

\section{DEVICE STRUCTURE AND WORKING PRINCIPLE}

Figure 1 show the device structure and its working principle of the simulated MD IPS LCD, where both the positive and negative dielectric anisotropy LC materials can be used. The rubbing direction is preferred to be along the vertical direction in Figure 1(a) and the LC directors are aligned homogeneously on the glass substrates. The chevron-shaped electrode is bent and has a bending angle, $\alpha$, which is set as the angle between the chevron arm extension direction and the vertical direction. A serial of chevron-shaped electrodes are alternatively arranged to form the inter-digital electrodes on the same substrate as the common electrode and the pixel electrode, respectively, which are connected to the thin-film transistors (TFTs) in the practical LCD devices.

The principal axis of the linear polarizer near the bottom substrate is parallel to the LC alignment (i.e., rubbing) direction. When there is no voltage applied, the incident light is completely blocked by the crossed polarizers, which results in a normally black state. When the applied voltage exceeds the threshold, the transversal electric fields are created and the electric field lines are in the parabolic form in the whole display area. Correspondingly, the LC directors with 

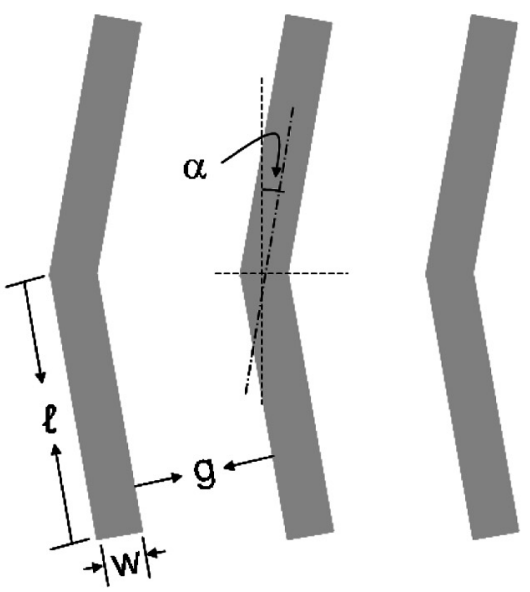

(a)

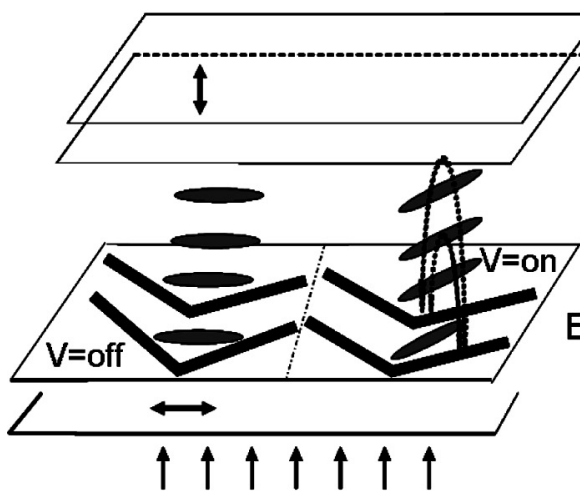

Analyzer

Top substrate

Bottom substrate

Polarizer

Backlight

(b)

FIGURE 1 The typical structure of MD IPS LCD (Fig. 1(a)) and its working principles (Fig. 1(b)).

positive (or negative) dielectric anisotropy between the electrodes would be reoriented along (or perpendicular) the electric field direction. The LC directors above two chevron arm regions are twisted into different directions so that the multi-domain LC configuration is generated and light transmits through the crossed polarizers.

During the simulation, we chose a chevron-shaped MD IPS LCD using a positive LC material (Merck: MLC 6692), where its physical properties are summarized as follows: $\gamma_{1}=0.1 \mathrm{~Pa} \cdot \mathrm{S}, \Delta \varepsilon=10.3, \mathrm{~K}_{11}=$ $9.6 \mathrm{pN}, \mathrm{K}_{22}=6.1 \mathrm{pN}, \mathrm{K}_{33}=14.1 \mathrm{pN}$ and $\Delta \mathrm{n}=0.085$ at $\lambda=550 \mathrm{~nm}$. 
The cell gap is $\mathrm{d}=4 \mu \mathrm{m}$, the width of the chevron-shaped electrode is $w=3 \mu \mathrm{m}$, the electrode gap is $g=8 \mu \mathrm{m}$, and the chevron arm length is $l=128 \mu \mathrm{m}$. The bending angle of the chevron-shaped electrodes, $\alpha$, is varied from $10^{\circ}$ to $45^{\circ}$. The employed polarizers are Nitto Denko HEG1425 types with a thickness of $210 \mu \mathrm{m}$. The maximum transmittance of the polarizer/analyzer pair is $35 \%$.

\section{RESULTS AND DISCUSSION}

\subsection{Voltage-dependent Transmittance}

Figure 2 plots the VT curves of the MD IPS cell at $\lambda=550 \mathrm{~nm}$ and different bending angles. As the bending angle increases, the maximum transmittance gradually decreases and the on-state voltage increases. For instance, the maximum transmittance occurs at $5 \mathrm{~V}_{\text {rms }}$ for $\alpha=10^{\circ}$, but increases to $7 \mathrm{~V}_{\mathrm{rms}}$ as the bending angle increases to $\alpha=40^{\circ}$. The higher on-state voltage is due to the required effective projected electric field to switch the LC directors when the bending angle of the chevron electrode increases. In the meantime, the threshold voltage is lowered from 1.25 to $0.75 \mathrm{~V}_{\text {rms }}$ as the bending angle is increased from at $\alpha=10^{\circ}$ to $40^{\circ}$. A small threshold voltage indicates that the device can be operated at a low driving voltage with less power consumption. Here, we select the voltage point at the maximum transmittance as the bright state driving voltage to calculate the isocontrast view angle contours.

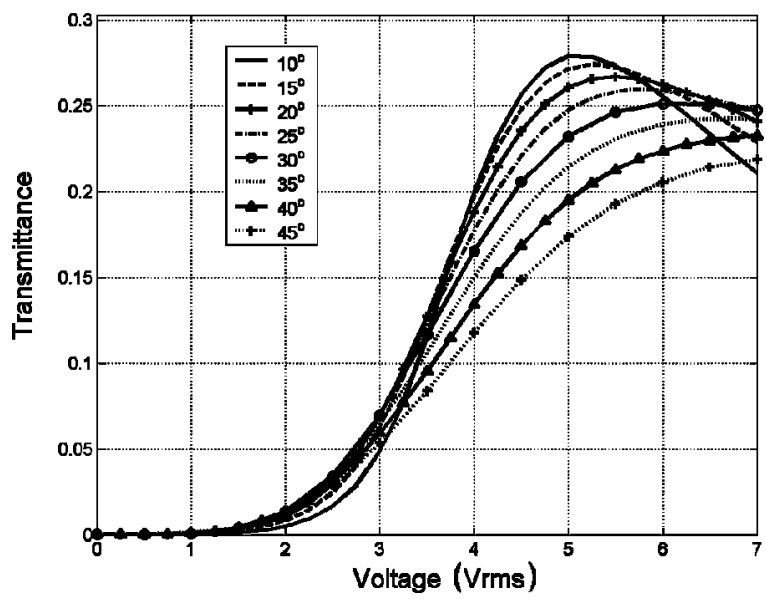

FIGURE 2 The voltage-dependent transmittance of the MD IPS mode with the different bending angles at the wavelength of $550 \mathrm{~nm}$. 


\subsection{Inherent Viewing Angle of MD IPS Mode}

Figure 3 plots the inherent isocontrast contour bars of the abovementioned MD IPS mode without any compensation films added.

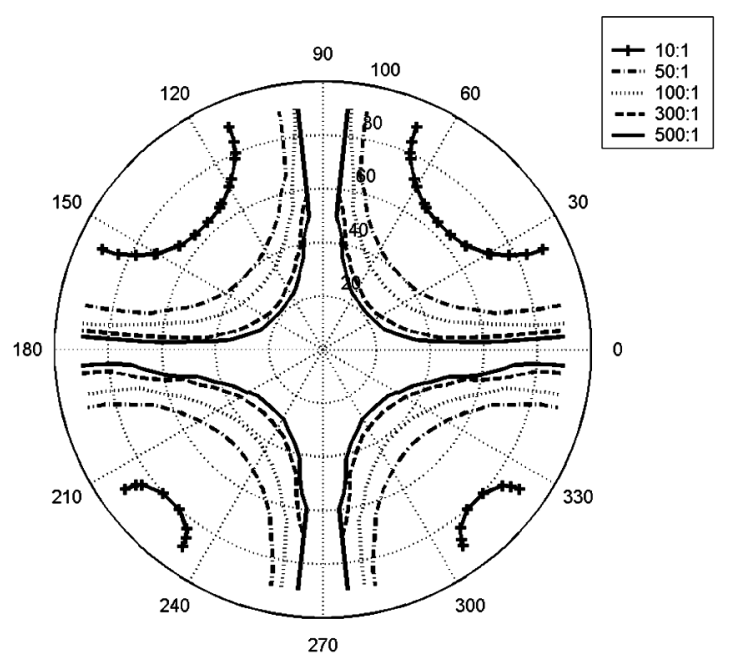

(a)

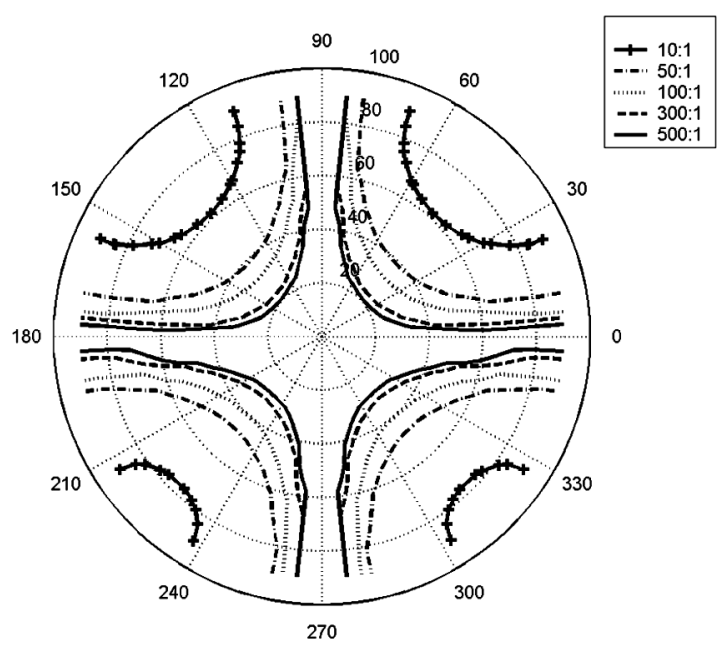

(b)

FIGURE 3 The inherent isocontrast contour bars of the MD IPS mode at the different bending angle, $\alpha$, and the wavelength of $550 \mathrm{~nm}$. Figure $3(\mathrm{a}): \alpha=10^{\circ}$ at $\mathrm{V}=5 \mathrm{~V}_{\mathrm{rms}} ;$ Figure $3(\mathrm{~b}): \alpha=30^{\circ}$ at $\mathrm{V}=6 \mathrm{~V}_{\mathrm{rms}}$. 
As the tilt angle departs from zero the iso-contrast bar becomes symmetric along the horizontal/vertical direction and the view angle is wide. For both the low bending angle of $10^{\circ}$ at $\mathrm{V}=5 \mathrm{~V}_{\mathrm{rms}}$ and the high bending angle of $30^{\circ}$ at $\mathrm{V}=6 \mathrm{~V}_{\text {rms }}$, their contrast ratios are all higher than 500:1 at $\pm 25^{\circ} \lambda=550 \mathrm{~nm}$. In the horizontal and vertical directions, the viewing angles extend all the way to $\pm 90^{\circ}$ with contrast ratio higher than 100:1. On the meanwhile, the 10:1 isocontrast view cones are limited at $\pm 75^{\circ}$ which are still not good enough for a practical LCD device. It is mainly from it is relatively high light leakage at the bisector plane of the polarizer transmission axes as shown in Figure 4. The inherent light leakage of the MD IPS mode without any compensation films is usually at the level of $5 \times 10^{-5}$, while the light leakages at the bisector plane of the polarizer transmission axes can reach $2 \times 10^{-4}$.

\subsection{Film Compensation for MD IPS Mode}

Chen et al. proposed to use a uniaxial A-plate and a C-plate to reduce the off-axis light leakage of the crossed polarizers and pointed out that the optical axis of the A-plate should align along the transmission axis of its adjacent polarizer [8]. The optimum film compensation principle has been successfully used in TN and VA LCDs. Here, we optimize the compensation films to widen the viewing angle of the IPS LCD using Chen's arrangement, in which a positive C-plate is placed after the LC cell and before the A-plate in-between the crossed polarizers. In the practical device assembly, these films are usually laminated together to minimize the interfacial reflections.

Poincaré sphere representation is a powerful means for analyzing the polarization state in each stage of a LCD. Figure 5 plots the Poincaré sphere representation of the film-compensated IPS LCD using a set of uniaxial positive C- and A-plates. From the Poincaré sphere, we can see that this kind of compensation film arrangement is to reduce the light leakage of the crossed polarizers at the oblique angle, especially when the obliquely viewing angle is in the bisect plane of the two polarizer transmission axes. Under this arrangement, the positive C-plate first moves the polarization state that exits through the analyzer from $P$ to $P^{\prime}$, then the positive A-plate further rotates the polarization state from $P^{\prime}$ back to the equator and meets at the polarizer's absorption point, $A b$. In this way, the polarization state $P$ can be converted back to $A b$ and the exit light is completely blocked by the analyzer. Therefore, the off-axis light leakage of the IPS LCD can be minimized. 


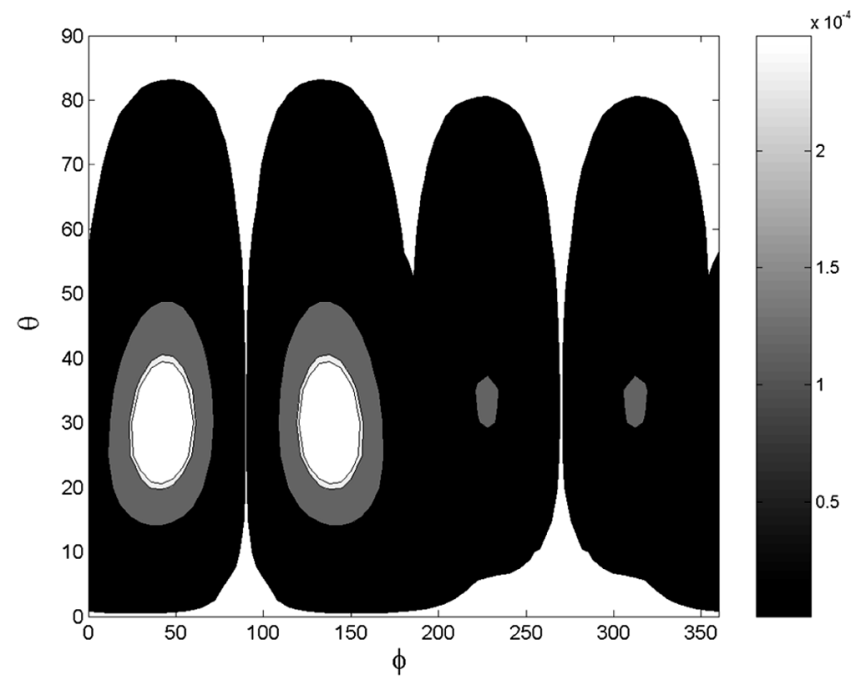

(a)

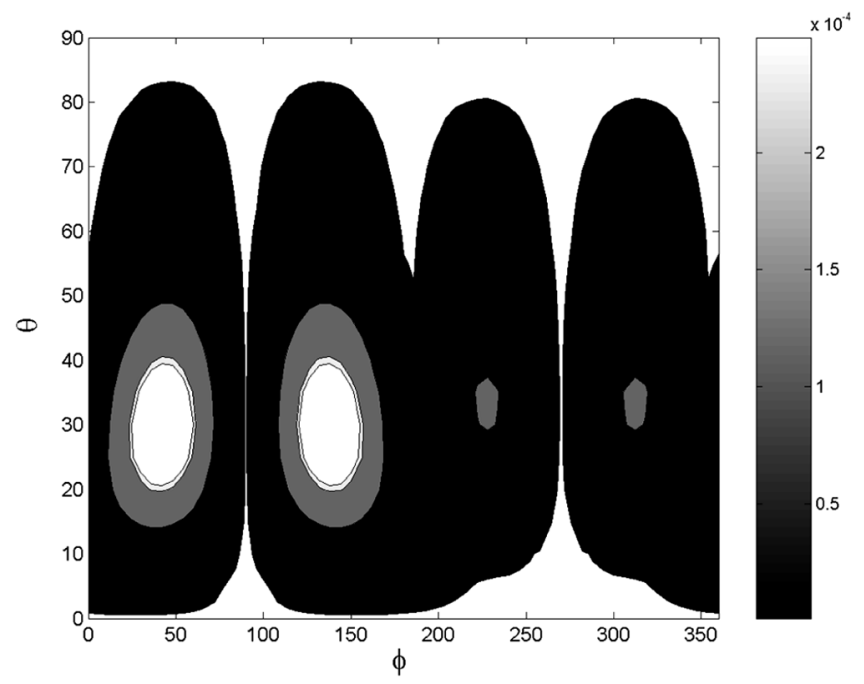

(b)

FIGURE 4 The inherent light leakage of the MD IPS mode at the different bending angle, $\alpha$, and the wavelength of $550 \mathrm{~nm}$. Figure $4(\mathrm{a}): \alpha=10^{\circ}$; Figure $4(b): \alpha=30^{\circ}$. 


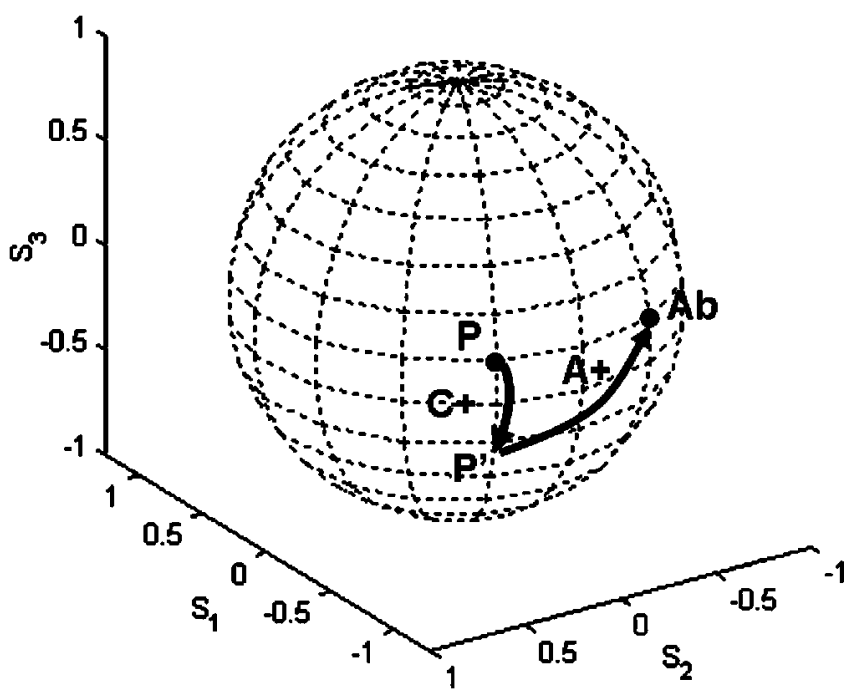

FIGURE 5 The principle of compensating the off-axis light leakage on the Poincaré sphere for MD IPS mode using a set of positive A-plate and C-plate.

\subsection{Viewing Angle of Film-compensated MD IPS Mode}

Through a searching calculation, we found that a positive C-plate with $d \cdot \Delta n=91.8 \mathrm{~nm}$, and an A-plate with $d \cdot \Delta n=155.4 \mathrm{~nm}$ are optional of eliminating the off-axis light leakage of the MD IPS mode. Figure 6 are the dark states of the film compensated MD IPS mode at the bending angle of $10^{\circ}$ and $30^{\circ}$, respectively. After adding the optimized uniaxial films, the light leakage can be reduced to as low as $6 \times 10^{-6}$, and at the level of $10^{-5}$ on the whole viewing range. Compared with the MD IPS mode without the compensation films, the film compensated MD IPS mode has 10 to 100 times improvement in eliminating the inherent light leakage from the crossed polarizers.

Figure 7(a) plots the isocontrast bar of the MD IPS mode using the proposed compensation films, where the bending angle is $10^{\circ}$ and the applied voltage is $5 \mathrm{~V}_{\text {rms }}$. Simulation results show that the 500:1 contrast ratio is extended to over $\pm 50^{\circ}$ viewing cone and the 100:1 contrast ratio is wider than $\pm 80^{\circ}$ viewing range. A similar result can also be obtained at a high bending angle of $30^{\circ}$ at the applied voltage of $6 \mathrm{~V}_{\text {rms }}$ as shown in Figure 7(b).

Under the practical circumstances, the film thickness may not be controlled precisely during the device fabrication process. Thus, it needs to consider the manufacturing tolerance. In the extreme cases, we assume the $d \cdot \Delta n$ values of the A-plate and C-plate are 


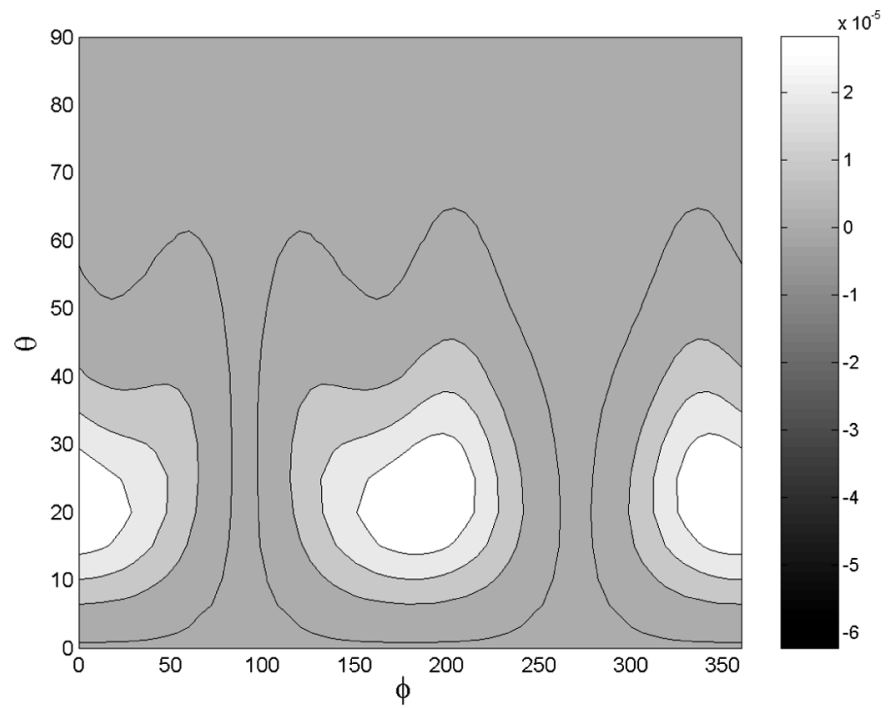

(a)

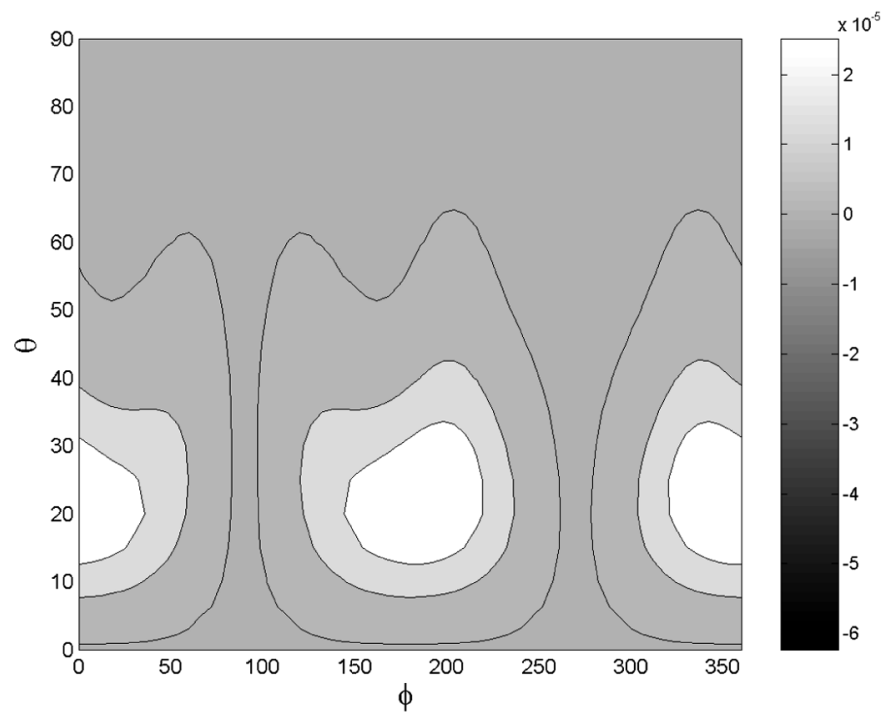

(b)

FIGURE 6 The light leakage of the MD IPS mode at the different bending angles using the optimized uniaxial films, where the positive C-plate and A-plate films have the respective $d \cdot \Delta n$ value of $91.8 \mathrm{~nm}$ and $155.4 \mathrm{~nm}$. Figure 6(a): $\alpha=10^{\circ}$; Figure 6(b): $\alpha=30^{\circ}$. 


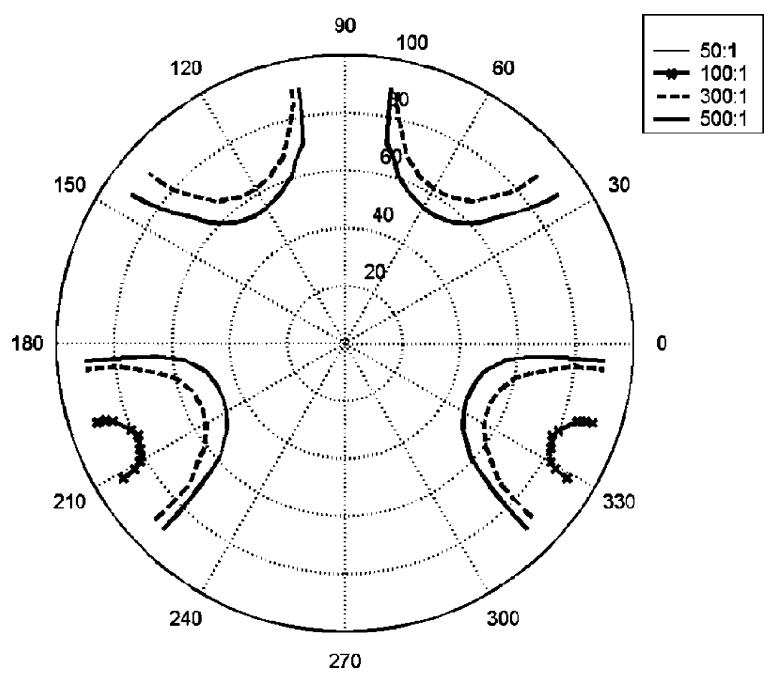

(a)

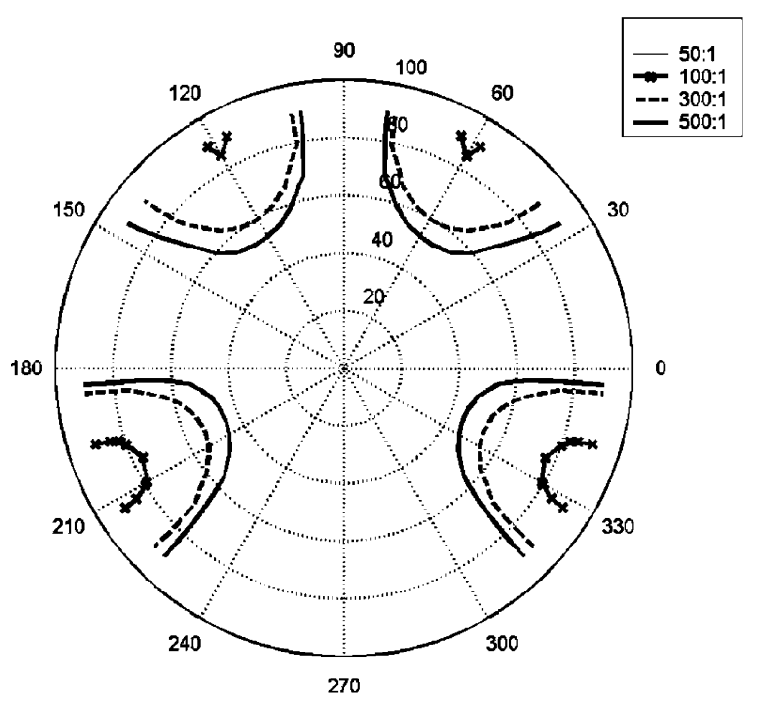

(b)

FIGURE 7 The isocontrast contour bars of the MD IPS mode at the different bending angles using the optimized uniaxial films, where the positive C-plate and A-plate films have the respective $d \cdot \Delta n$ value of $91.8 \mathrm{~nm}$ and $155.4 \mathrm{~nm}$. Figure $7(\mathrm{a}): \alpha=10^{\circ}$ at $\mathrm{V}=5 \mathrm{~V}_{\text {rms }}$; Figure $7(\mathrm{~b}): \alpha=30^{\circ}$ at $\mathrm{V}=6 \mathrm{~V}_{\mathrm{rms}}$. 


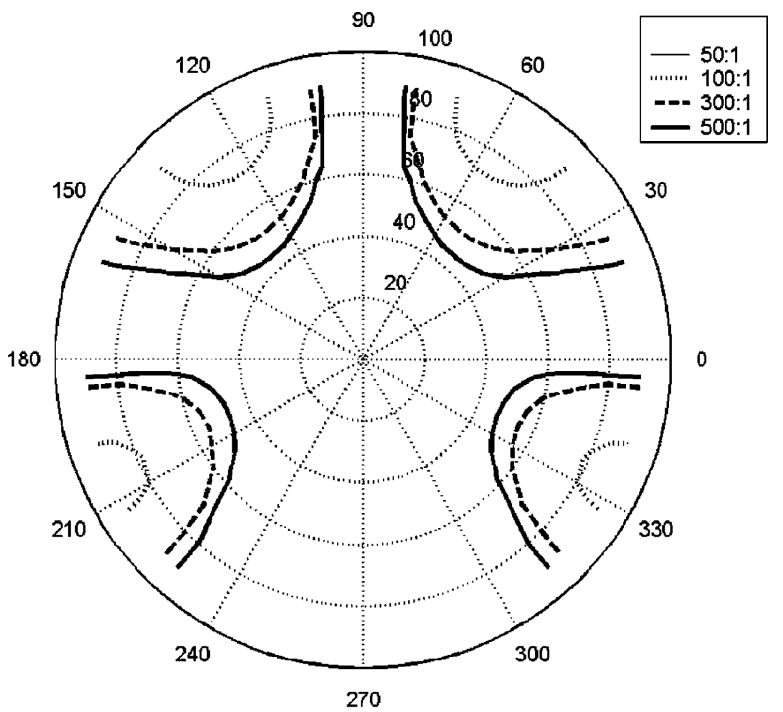

(a)

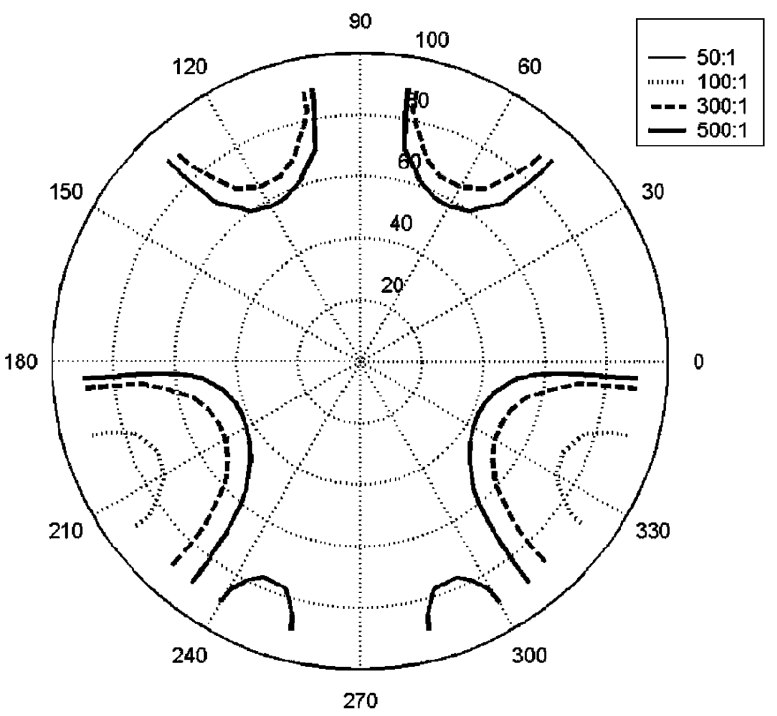

(b)

FIGURE 8 The manufacturing tolerance on the isocontrast bar of the MD IPS mode using the optimized uniaxial films, where the positive C-plate and A-plate films have the respective $d \cdot \Delta n$ value of $91.8 \mathrm{~nm}$ and $155.4 \mathrm{~nm}$. The bending angle is $30^{\circ}$ and the applied voltage is $6 \mathrm{~V}_{\mathrm{rms}}$. Figure $8(\mathrm{a}): d \cdot \Delta n$ of the C-plate and A-plate are both $5 \%$ below the optimal values; Figure $8(\mathrm{~b})$ : $d \cdot \Delta n$ of the C-plate and A-plate are both $5 \%$ above the optimal values. 
simultaneously $5 \%$ below and above their optimal values. As an example, the results for the MD IPS mode at the bending angle of $30^{\circ}$ and the applied voltage of $6 \mathrm{~V}_{\mathrm{rms}}$ are plotted in Figures $8(\mathrm{a})$ and (b), respectively. In both cases, the viewing angle is slightly narrowed, but their contrast ratio still maintains $\sim 100: 1$ at $\pm 70^{\circ}$ viewing cone. These results are very promising as a wide viewing LCD device. Therefore, a MD IPS mode LCD with enhanced viewing angle can be realized using the optimized uniaxial compensation films.

\section{CONCLUSIONS}

The electro-optic performance of a MD IPS LCD with chevron electrode is characterized with the different electrode bending angles. The viewing angle of the MD IPS LCD is enhanced using a set of optimized uniaxial C- and A-plate compensation films, where the contrast ratio can reach at 100:1 on the whole viewing range. The wide viewing advantage of the MD IPS LCD is helpful for its potential applications in high quality LCD TVs and computer monitors.

\section{REFERENCES}

[1] Wu, S. T. \& Yang, D. K. (2001). Reflective Liquid Crystal Displays, Wiley: New York, USA.

[2] Koike, Y. \& Okamoto, K. (1999). Fujitsu Sci. Tech. J., 35, 221.

[3] Kim, S. (2005). SID'05 Digest, 36, 1842.

[4] Ishii, Y., Mizushima, S., \& Hijikigawa, M. (2001). SID’01 Digest, 1090.

[5] Kiefer, R., Webber, B., Windscheid, F., \& Baur, G. (1992). Japan Displays'92, 547.

[6] Oh-e, M. \& Kondo, K. (1995). Appl. Phys. Lett., 67, 3895.

[7] Oh-e, M., Yoneya, M., \& Kondo, K. (1997). J. Appl. Phys., 82, 528.

[8] Chen, J., Kim, K., Jyu, J., Souk, J., Kelly, J., \& Bos, P. (1998). SID’98 Digest, 29 , 315.

[9] Saitoh, Y., Kimura, S., Kusafuka, K., \& Shimizu, H. (1998). Jpn. J. Appl. Phys., 37, 4822.

[10] Aratani, S., Klausmann, H., Oh-e, M., Ohta, M., Ashizawa, K., Yanagawa, K., \& Kondo, K. (1997). Jpn. J. Appl. Phys., 36, L27.

[11] Klausmann, H., Aratani, S., \& Kondo, K. (1998). J. Appl. Phys., 83, 1854.

[12] Wu, S. T. (1995). SID Tech. Digest, 26, 555.

[13] Lu, R., Zhu, X., Wu, S. T., Hong, H., \& Wu, T. X. (2005). J. Display Tech., 1, 3.

[14] Lu, R., Zhu, S. T., Hong, H., \& Wu, T. X. (2005). SID’05 Digest, 36, 654-657.

[15] Lien, A. (1990). Appl. Phys. Lett., 57, 2767.

[16] Pasqual, F., Deng, H., Fernandez, F., Day, S., Davies, J., Johnson, M., Put, A., Eerenbeemd, J., Haaren, J., \& Chapman, J. (1999). IEEE Trans. Electron Devices, 46,661 . 